\title{
Non-repudiation in order, delivery and payment process for a sustainable online business
}

\begin{abstract}
Online businesses in Malaysia suffer from the lack of non-repudiation in the ordering, delivery and payment process. Most businesses cannot sustain their growth because of this limitation. The paper presents a new model for the implementation of online business. The solutions can also be adapted to any country for the most part except for the delivery process that requires the use of a smartcard-based national identity card. The non-repudiation evidences that are generated from the system can be used by both the consumer and the merchant to resolve possible future disputes. The evidence is also admissible in the court of law since they are generated from the Public Key Infrastructure (PKI) that is recognized by the authority as legally binding.
\end{abstract}

Keyword: Non-repudiation; Online business; Cybersecurity; Security framework 